\title{
Discrete Device
}

National Cancer Institute

\section{Source}

National Cancer Institute. Discrete Device. NCI Thesaurus. Code C49924.

A device that exists separate from other devices; can refer to electronic components

that are not part of an integ rated circuit. 